\title{
INDOCYANINE GREEN ANGIOGRAPHIC FINDINGS IN CENTRAL SEROUS CHORIORETINOPATHY
}

\author{
FELICE CARDILLO PICCOLINO, LUIGI BORGIA, EDOARDO ZINICOLA and \\ MARIO ZINGIRIAN \\ Genoa, Italy
}

\begin{abstract}
SUMMARY
The purpose of this study is to better characterise, on the basis of a large number of cases and follow-up evaluations, choroidal abnormalities recently observed with indocyanine green (ICG) angiography in central serous chorioretinopathy (CSC). Digital ICG videoangiography was performed in 145 patients with active or inactive, acute or chronic CSC. Forty-eight patients were re-examined in a follow-up period of 6-22 months (mean 10 months). Areas of choroidal leakage attributable to hyperpermeability of the choriocapillaris were found in $98.6 \%$ of patients in association with active or resolved pigment epithelial leaks and pigment epithelial detachments. Diffusion of ICG into the choroid was characterised by rapid centrifugal spreading of the dye with a wash-out pattern which was particularly evident in areas corresponding to pigment epithelial detachments. In patients with a long-standing disease, when choroidal hyperfluorescence faded, hypofluorescent spots became increasingly evident revealing pigment epithelial alterations not shown by fluorescein angiography. Areas of choroidal leakage remained unchanged in each patient during the followup period, even when subretinal exudation resolved either spontaneously or after photocoagulation. In 5 eyes we observed the appearance of leakage points on pre-existing areas of choroidal leakage. Zonal hyperpermeability of the choriocapillaris characterises all the evolutional stages of CSC and seems to be the primary alteration of this disease. When it corresponds to pigment epithelial detachments choriocapillaris hypermeability is probably associated with local hyperperfusion.
\end{abstract}

Modern indocyanine green (ICG) angiography, using high-resolution digital imaging procedures, has to a great extent overcome the limits of

From: Department of Ophthalmology, University of Genoa, Genoa, Italy.

Correspondence to: F. Cardillo Piccolino, MD, Clinica Oculistica dell'Università, Ospedale S. Martino, Pad. 9, Viale Benedetto XV, 5-16132 Genoa, Italy. fluorescein angiography in imaging of the choroidal vasculature. $^{1-3}$ The first important result of introducing this new tool of clinical investigation is its better angiographic delineation, and thus greater possibilities for laser treatment of the occult choroidal neovascularisation in age-related macular degeneration. ${ }^{2,4}$ But ICG angiography also seems able to provide a better understanding of various other chorioretinal disorders. It could be the right tool to clarify the role of the choroid in central serous chorioretinopathy (CSC).

This disease is characterised by a serous retinal detachment in the posterior pole and one or more focal leaks revealed by fluorescein angiography at the level of the retinal pigment epithelium. It essentially affects young and middle-aged men, usually without remarkable medical histories. The course of the disease is benign for the majority of the patients, but recurrences and chronic persistence of subretinal fluid can lead to chorioretinal atrophic changes with permanent visual loss. ${ }^{5}$

Despite good knowledge of the clinical and fluorescein angiographic characteristics, the aetiology and the pathophysiological basis of the disease are still unknown. In recent years attention has been focused on the role of the retinal pigment epithelium, ${ }^{6,7}$ but the observations with ICG angiography by our group and others suggest a primary involvement of the choroid in CSC. ${ }^{8-11}$ Schedier et al. ${ }^{9}$ observed perfusion defects of the choroid in $63 \%$ of their patients with CSC and stressed the role of this alteration in the pathogenesis of the disease. However, choroidal perfusion defects were not recognised with ICG angiography in our first series of patients and in the series of Guyer et al. ${ }^{10,11}$ The main result of our previous study was the constant observation of areas of dye diffusion in the choroid corresponding to the pigment epithelial alterations that characterise $\mathrm{CSC} .{ }^{10}$ We proposed zonal hyperpermeability of the choriocapillaris as the primary 


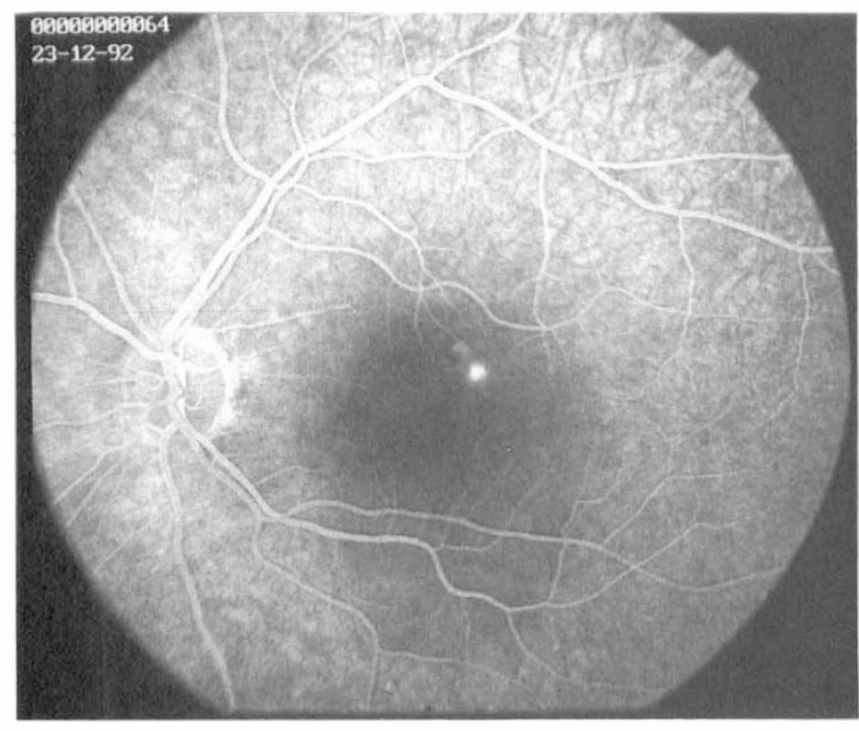

(a)

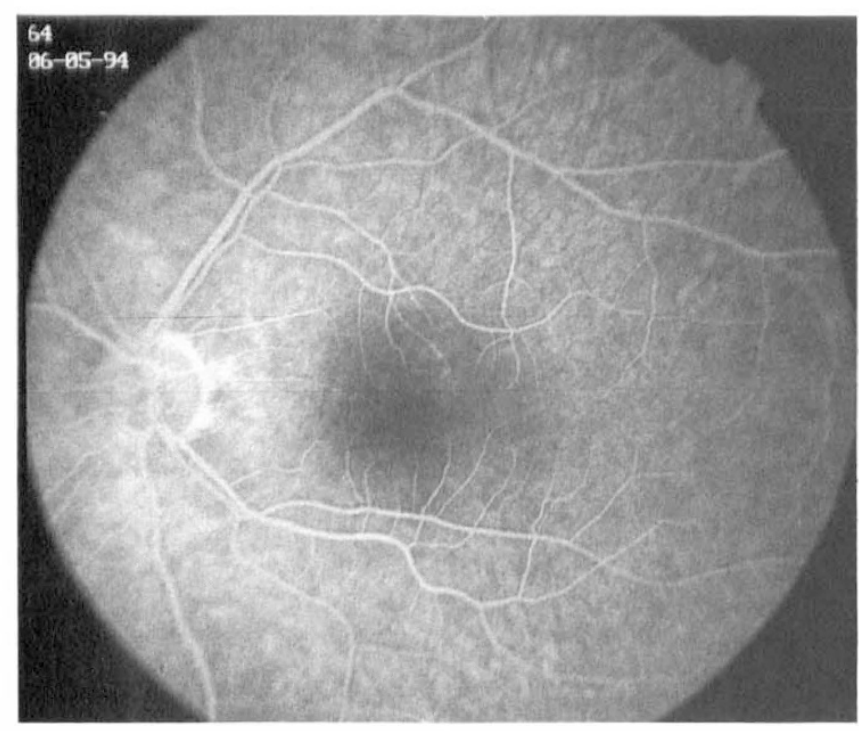

(c)

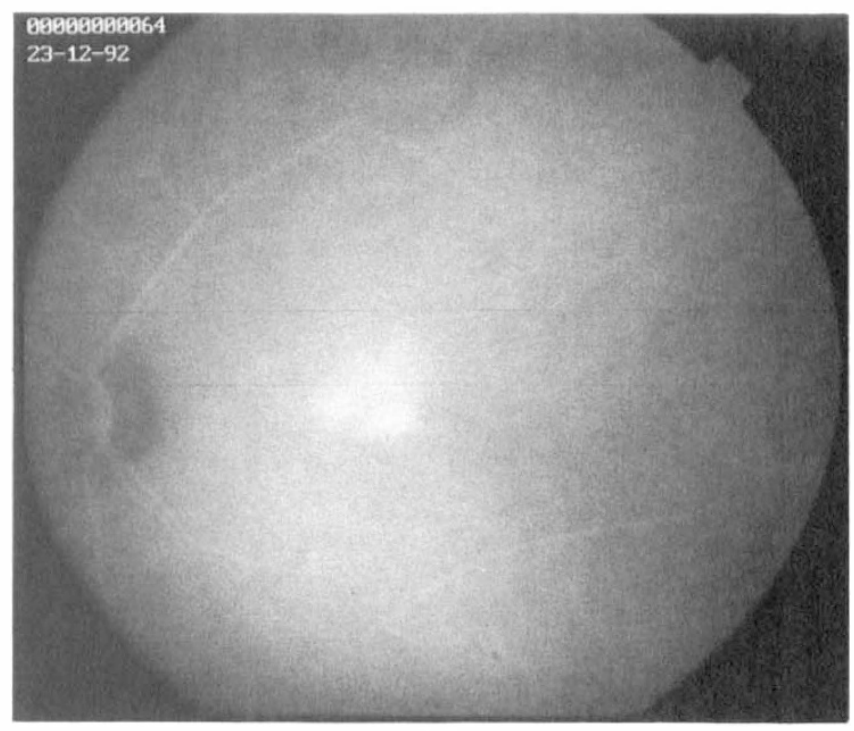

(b)

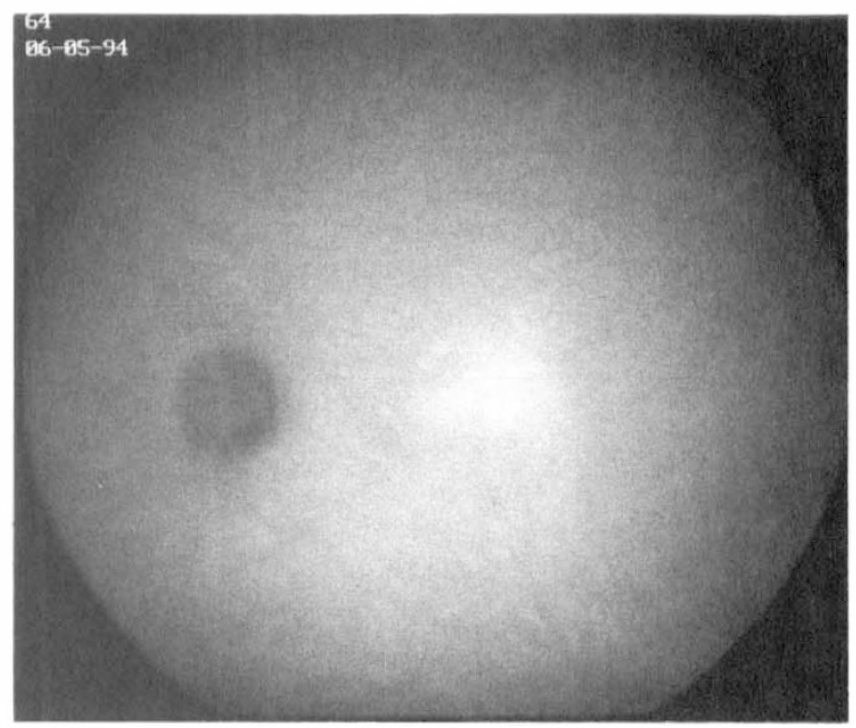

(d)

Fig. 1. Central serous chorioretinopathy with resolution of the subretinal but not of the choroidal exudation. (a), (b) Fluorescein and indocyanine green (ICG) photographs taken at the initial examinaton. (c), (d) Fluorescein and ICG photographs taken about 11/2 years later.

lesion in CSC, leading to degenerative alterations of the retinal pigment epithelium and passage of fluid in the subretinal space. ${ }^{10}$

The purpose of this report is: (1) to verify the results of our previous study analysing the ICG angiographic findings in a large number of patients with CSC; $(2)$ to study the evolution of the ICG angiographic pattern in CSC.

\section{PATIENTS AND METHODS}

A total of 145 patients with active or inactive CSC, examined in the past 2 years at the Retina Service of the University Eye Clinic of Genoa, were included in this study. Exclusion criteria were: (1) a history of ocular trauma; (2) vascular, inflammatory and tumoral manifestations of the fundus known to be associated with subretinal fluid and chorioretinal scars; (3) macular drusen; (4) pigment epithelial detachments not associated with leakage points at least in one eye; (5) clinical or fluorescein angiographic signs of choroidal neovascularisation. Thirtythree of the 34 patients reported in our previous study on CSC and ICG angiography were included in the present series. ${ }^{10}$ The patient excluded was found to have a small choroidal neovascularisation which had simulated CSC. ${ }^{10}$ On the basis of clinical history, fundus examination and fluorescein angiography the cases were classified as acute CSC (85), healed CSC (12) and chronic CSC (48). Chronic CSC was defined as cases in which widespread pigment epithelial alterations could be ascribed to persistent or recurrent subretinal exudation. ${ }^{12,13}$ 


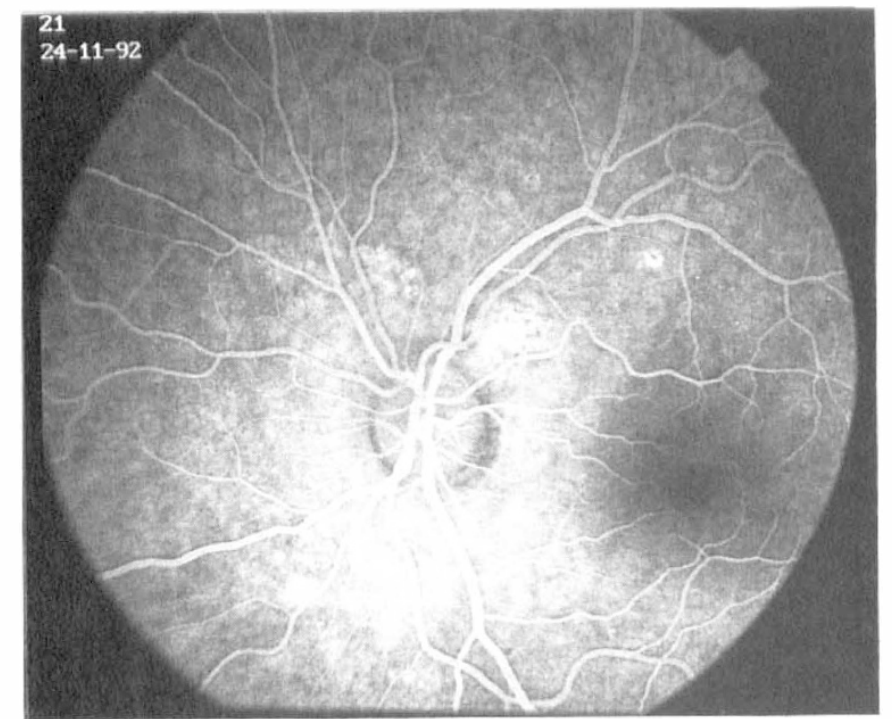

(i)

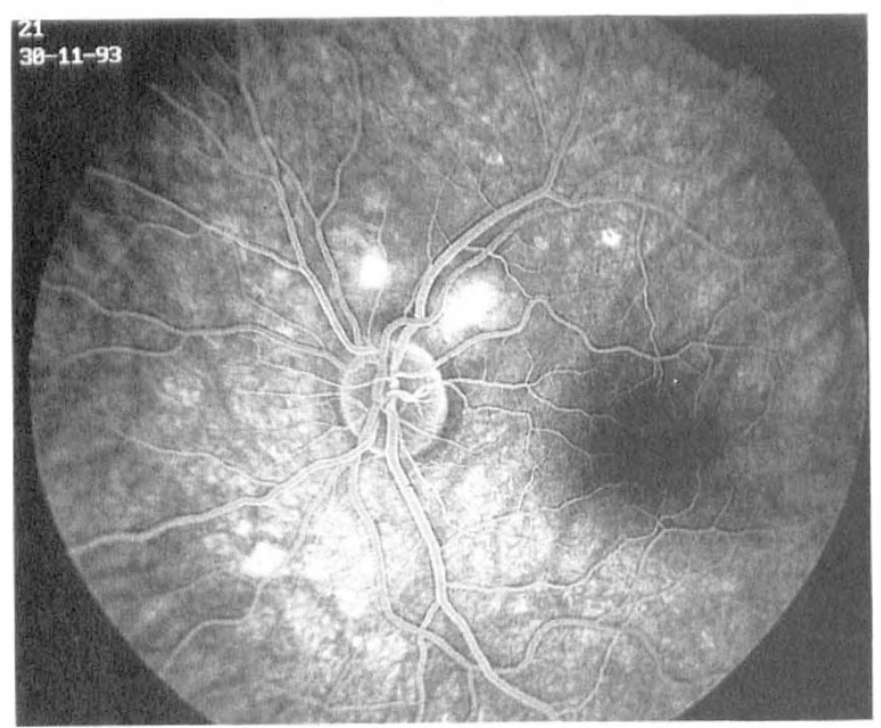

$(c)$

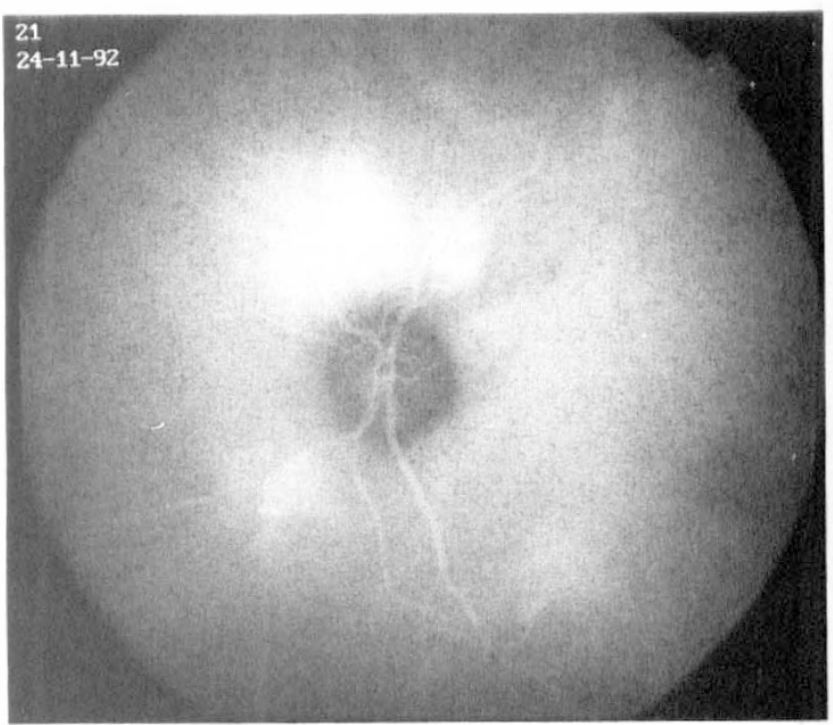

(1)

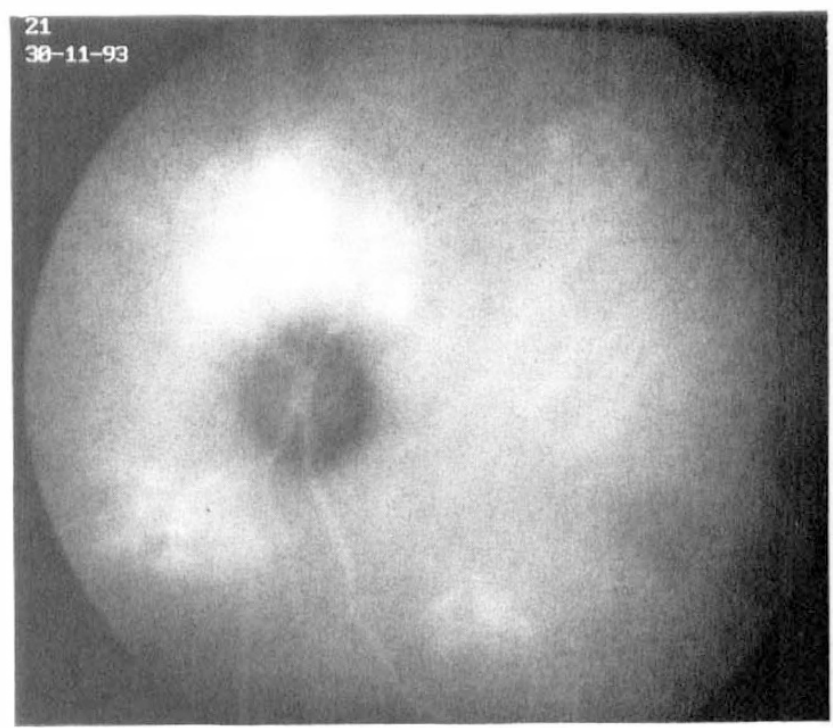

(d)

Fig. 2. Central serous chorioretinopathy with a leakage point corresponding to a pre-existing area of choriocapillaris hyperpermeability. (a), (b) Fluorescein and indocyanine green (ICG) photographs taken at the initial examination. (c), (d) Fluorescein and ICG photographs taken 1 year later.

Each patient underwent digital ICG videoangiography using the Topcon IMAGEnet H1024 Digital Imaging System (Ijssel, The Netherlands). For this examination one-third of a $10 \mathrm{ml}$ solution containing $50 \mathrm{mg}$ of ICG was injected intravenously. ICG images were taken at 1 or 2 second intervals until the choroid was completely filled, and thereafter images were obtained at intervals of increasing length until 30-60 minutes after injection. Fortyeight patients, of whom 32 had acute CSC and 16 had chronic CSC, were re-examined every 3-6 months in a follow-up period of 6-22 months (mean 10 months). Fifteen of these patients received laser photocoagulation of the leakage points.

On the ICG angiograms we evaluated choroidal filling, choroidal vascular permeability and altera- tions of the retinal pigment epithelium. We defined as perfusion defects of the choroid areas of hypofluorescence persisting when the surrounding choroidal veins were completely filled. In the areas of choroidal leakage we evaluated the characteristics of dye diffusion in the angiographic sequence. We recognised a 'wash-out phenomenon' where the ICG dye was cleared from the stained choroidal tissue with a centrifugal movement in the space of a few minutes. In patients with follow-up we looked for any changes relative to the ICG angiographic findings obtained at the first examination.

\section{RESULTS}

One hundred and thirty-two of the 145 patients were male. The average age was 40.8 years (range 29-56 


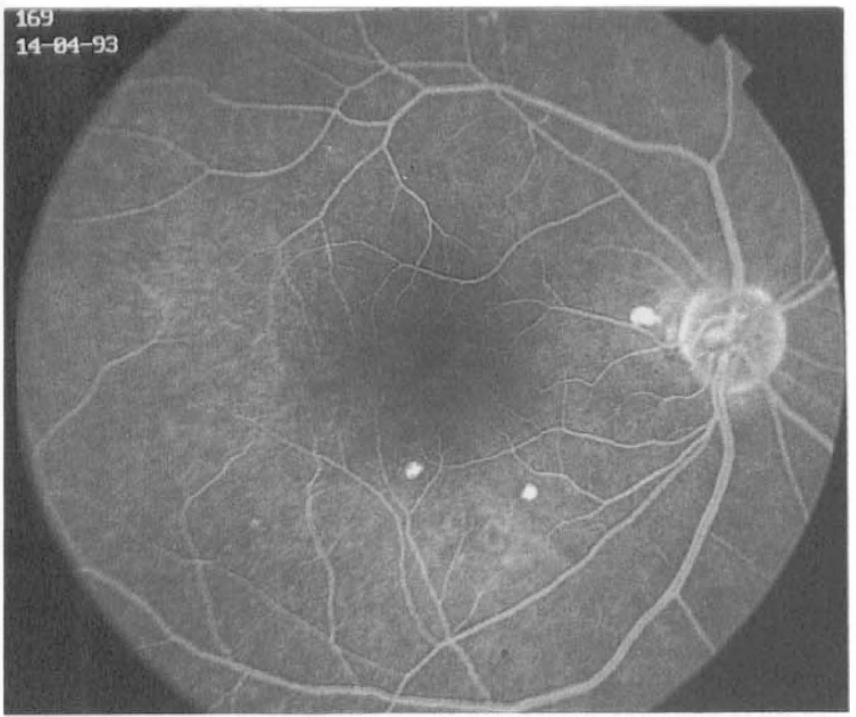

(a)

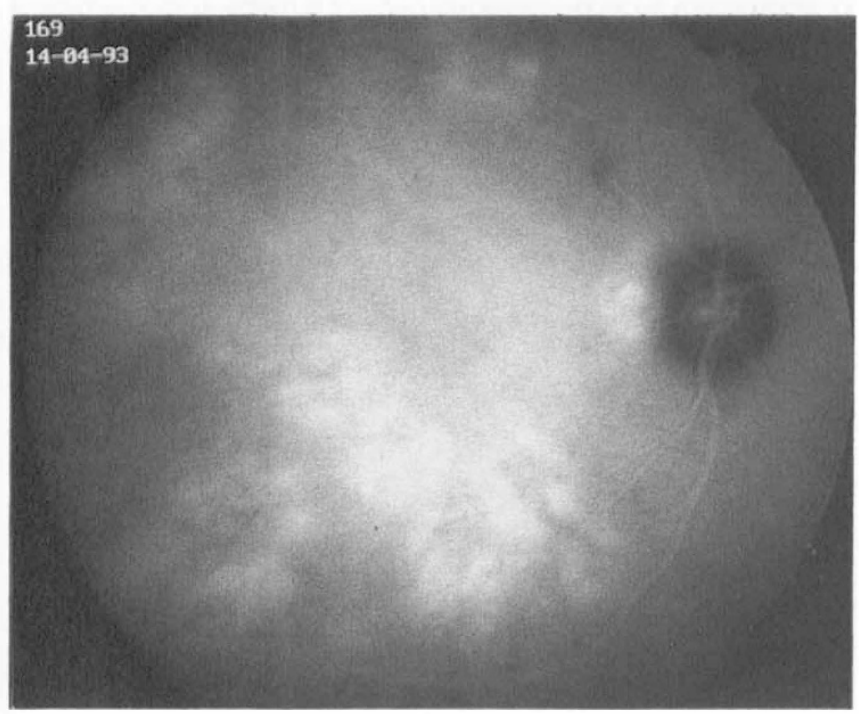

(c)

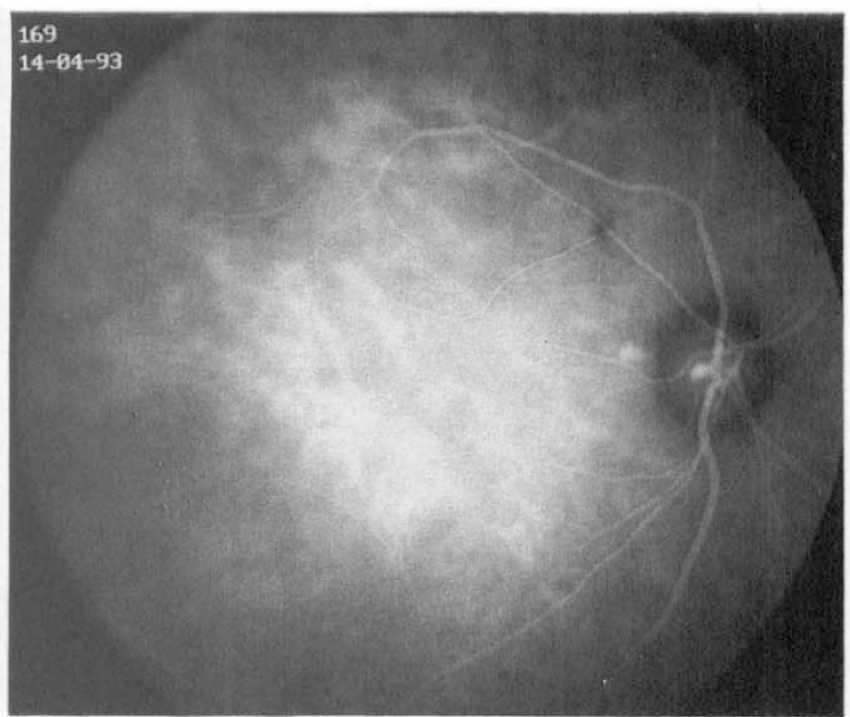

(b)

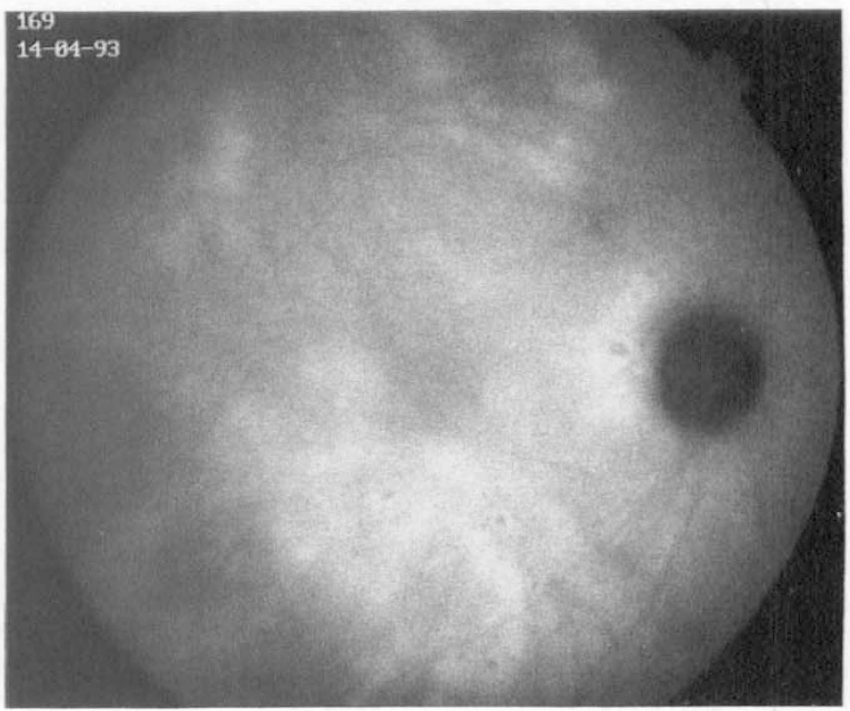

(d)

Fig. 3. (a) Fluorescein photograph showing small pigment epithelial detachments in a patient with central serous chorioretinopathy in the fellow eye. (b) Indocyanine green staining of the detachments and of underlying choroidal areas. (c), (d) Progressive centrifugal shifting of the dye in the choroid with washout of previously fluorescent areas and appearance of dark spots.

years) for patients with acute or healed CSC and 52.4 years (range 34-66 years) for patients with chronic CSC. Patients with acute CSC had one or more leakage points, with different degrees of activity, in one (78) or both (7) eyes. The patients classified as having healed CSC had had an acute episode of CSC less than a year before, which had resolved spontaneously or following laser treatment.

The patients with chronic CSC complained of visual impairment of more than 2 years' duration. Visual acuity varied considerably: from $20 / 20$ to less than 20/200. One half of the patients had a visual acuity between $20 / 30$ and $20 / 50$ in the worse eye. Flourescein angiography revealed bilateral pigment epithelial changes in all these patients. Large irregular zones of pigment epithelial atrophy or vertically oriented atrophic tracts were present in at least one eye. Thirty-two patients had subretinal fluid with pigment epithelial leakage or decompensation. In the remaining cases episodes of active CSC were documented by previous examinations.

Small pigment epithelial detachments were present in $40(27.5 \%)$ patients: in $15(17.6 \%)$ with acute and in $25(52 \%)$ with chronic CSC. Some of the smallest pigment epithelial detachments were recognised only by their characteristic imaging on the ICG angiograms.

During the follow-up period subretinal exudation and fluorescein leakage points disappeared in the 15 eyes that underwent photocoagulation and in another 12 eyes that had not been treated. In 5 


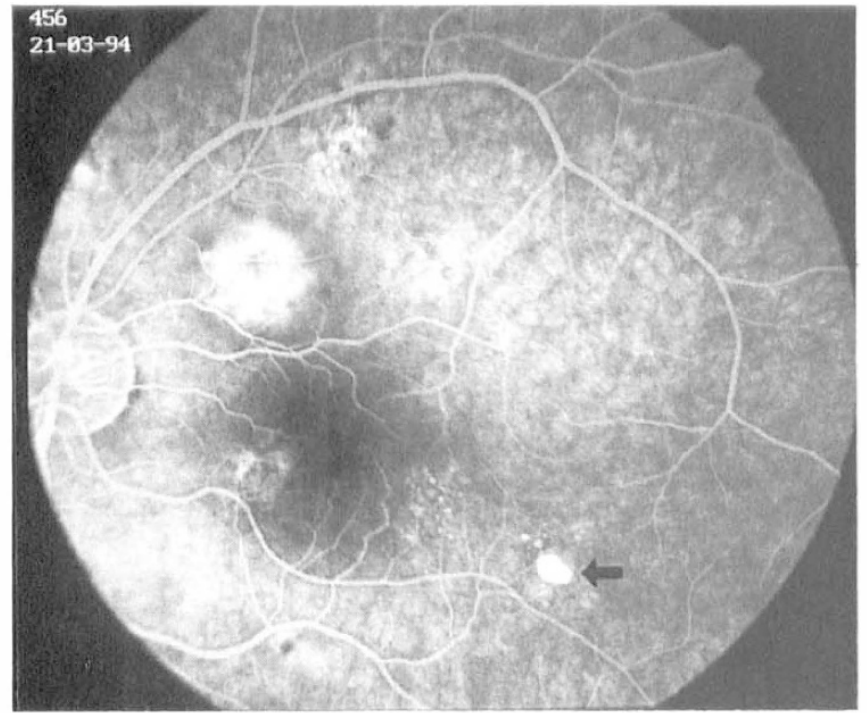

(i)

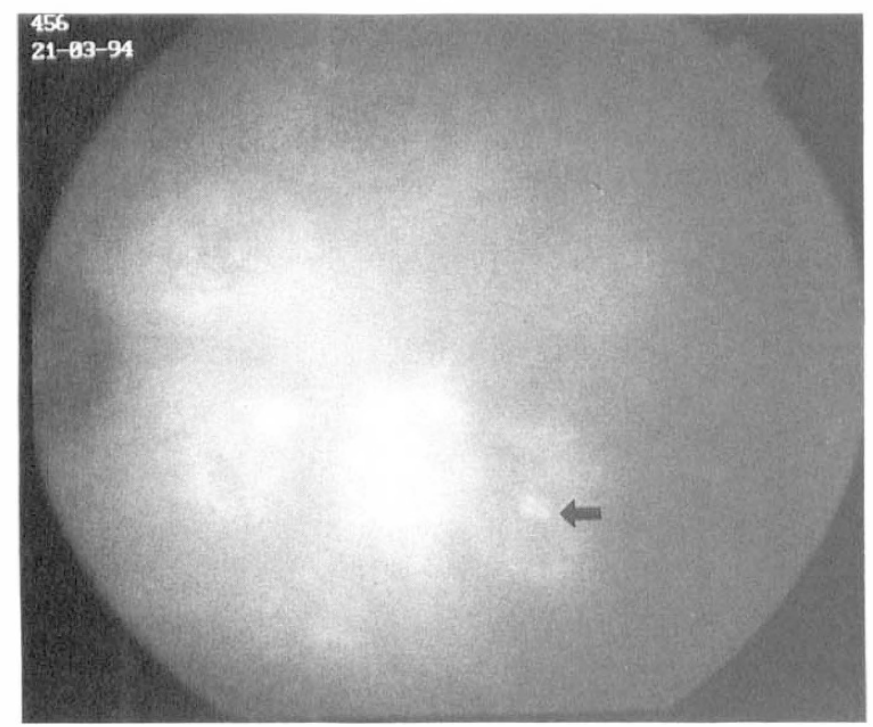

(c)

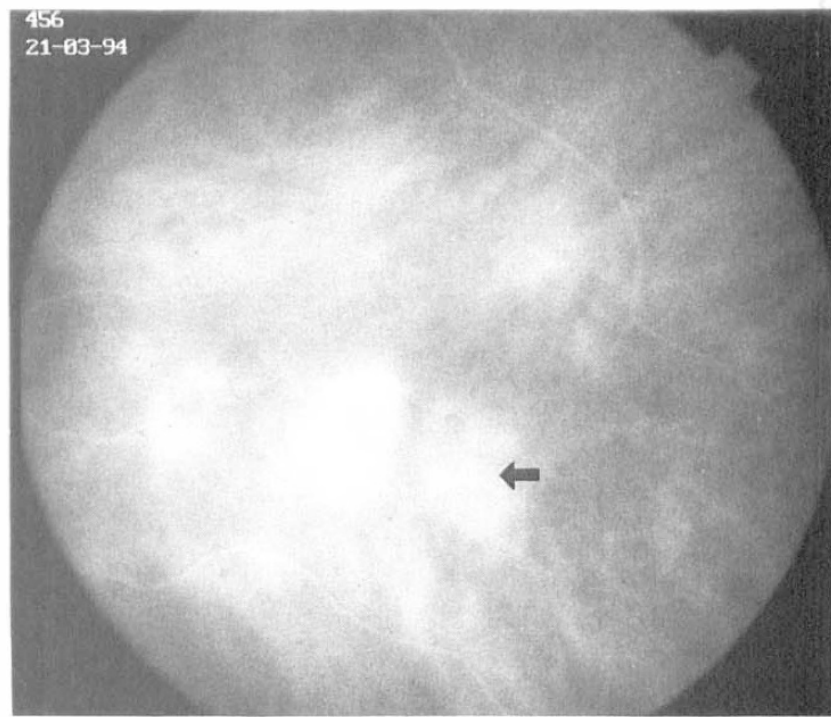

(b)

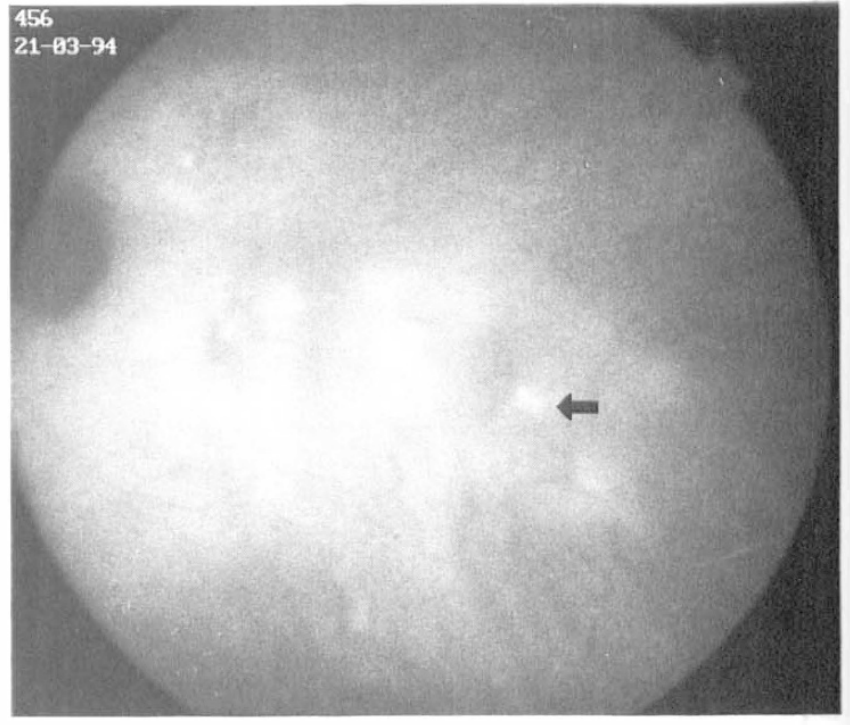

(d)

Fig. 4. (a) Fluorescein photograph in an eye with central serous chorioretinopathy and diffuse alterations of the retinal pigment epithelium. The arrow indicates a pigment epithelial detachment. (b)-(d) The indocyanine green angiographic sequence shows the wash-out phenomenon in the areas of choridal leakage, in particular in areas corresponding to the pigment epithelial detachment (arrow).

patients new leakage points were found during the follow-up examinations.

The behaviour of ICG at the leakage points was essentially analogous to that of fluorescein (Figs. 1,2). When fluorescein leakage was low the subretinal diffusion of ICG could hardly be distinguished from the background fluorescence. In the late phase of ICG angiography, when background fluorescence was considerably reduced, a hyperfluorescent spot could be seen at the level of the pigment epithelial defects, as well as a diffuse staining of the subretinal fluid. The detachments of the retinal pigment epithelium became fluorescent in the first minutes of the ICG examination. The dye was then gradually eliminated from the subepithelial space leaving residues along the border of the detachment (Figs.
$3,4)$. In the more advanced angiographic phases the pigment epithelial detachments appeared as hypofluorescent areas surrounded by a hyperfluorescent ring.

We observed defects of choroidal perfusion in 4 patients $(2.7 \%), 2$ of whom suffered from hypertension. All had multiple pigment epithelial alterations. In each case more areas of delayed choroidal filling were observed, but filling was always completed during the recirculation phase. One or more areas of dye diffusion in the choroid became evident from 3 to 5 minutes after dye injection in all patients except two $(98.6 \%)$ with inactive disease (Figs. 1-4). This finding was observed beneath all leakage points, all areas of pigment epithelial decompensation and all pigment epithelial detachments. They also occurred 


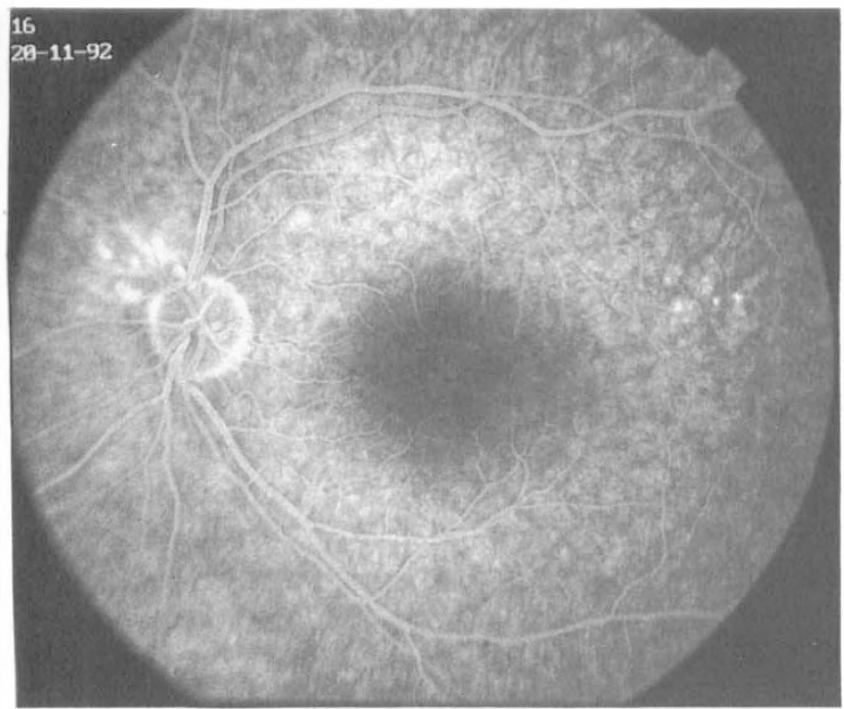

(a)

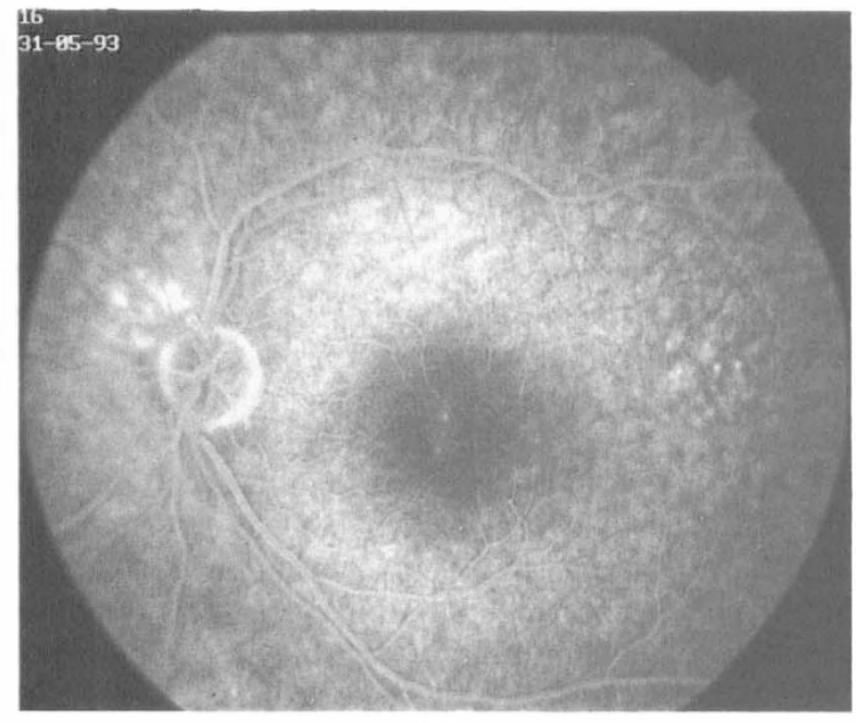

(c)

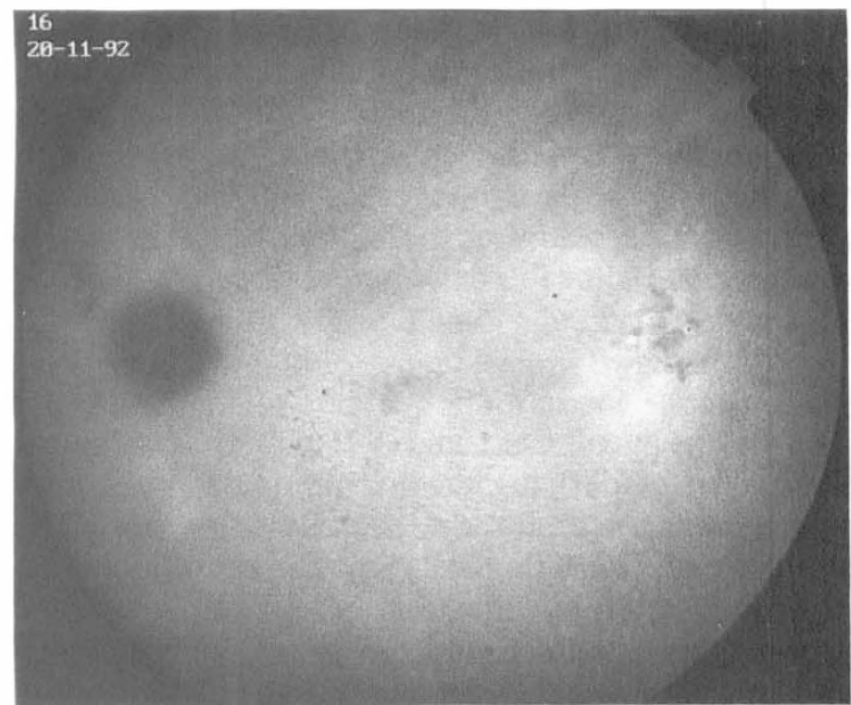

(b)

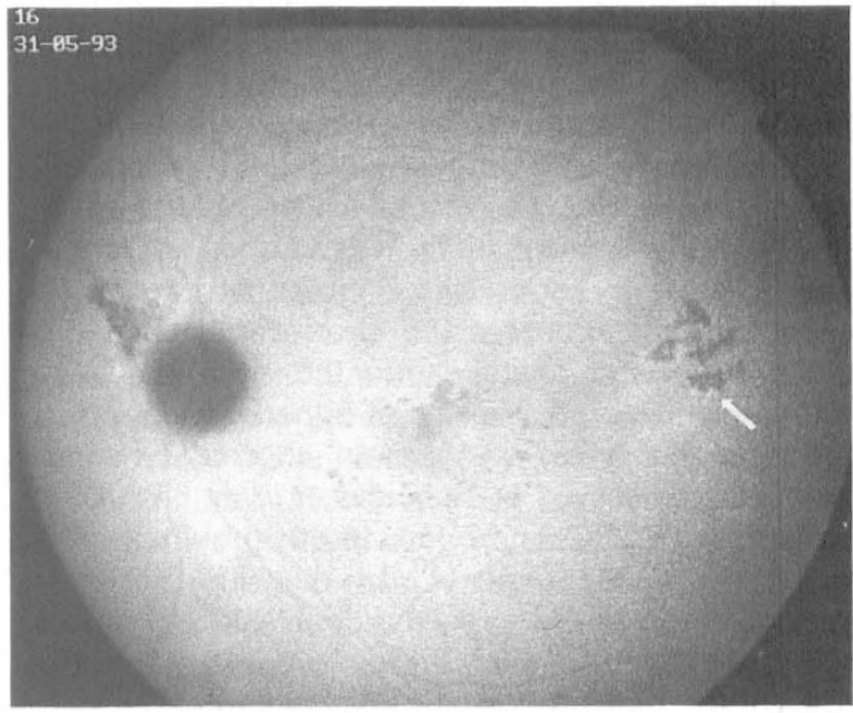

(d)

Fig. 5. (a)-(d) Enlargement of hypofluorescent spots evidenced by indocyanine green angiography (b, $d)$ in a patient with central serous chorioretinopathy. The fluorescein angiographic pattern is the same at the first observation (a) and 6 months later (c).

in some regions where degenerative changes of the retinal pigment epithelium were visible with fluorescein angiography and/or biomicroscopy. In the eyes with choroidal perfusion defects the areas of dye diffusion in the choroid did not show a clear topographic correspondence with the areas of delayed filling of the choriocapillaris.

Dye diffusion progressed in a centrifugal way from the area first stained, and in 2-5 minutes extended over double this area. Shifting of ICG in the choroid was particularly evident and rapid, with the wash-out phenomenon, corresponding to all the pigment epithelial detachments and several areas where alterations of the retinal pigment epithelium appeared as dark spots during the most advanced angiographic phases (Figs. 3, 4). In many areas of choroidal leakage, as background fluorescence faded, these hypofluorescent spots of varying size and irregular shape became more and more visible. They were more evident and numerous in patients with recurrent or chronic disease.

In the 48 cases followed with periodic examinations the areas of dye diffusion in the choroid were always present and did not show significant variations. They persisted also when the leakage points had disappeared spontaneously or after photocoagulation (Fig. 1). During follow-up we did not detect any areas of choroidal leakage which had not been present at the first observation. In the patients in whom new leakage points appeared, these corresponded to choroidal areas where dye diffusion was already evident at the first angiographic examination 
(Fig. 2). At the follow-up examinations hypofluorescent spots were unchanged in all cases except for 3 in which some patches appeared slightly enlarged (Fig. 5). In one eye we progressively observed the presence of a leak, of a pigment epithelial detachment after regression of the leak and of dark spots after regression of the detachment.

\section{DISCUSSION}

The aetiology of CSC and the pathophysiological mechanism underlying the formation of the serous retinal detachment that characterises this disease are still unclear. The hypothesis that the subretinal fluid originates from a previous choroidal exudative process dates back nearly 30 years. ${ }^{14}$ Fluorescein angiography, however, because of the limitation in imaging through the retinal pigment epithelium and the physiological leakage of fluorescein from the choriocapillaris, was not able to reveal choroidal vascular hyperpermeability in CSC. ICG angiography, which utilises a dye fluorescent in the nearinfrared range and almost completely bound to plasmatic proteins, has recently been able to reveal this choroidal abnormality. ${ }^{8-11}$ ICG does not leak in angiographically appreciable quantities from the normal fenestrations of the choriocapillaris; ${ }^{15}$ thus detection of dye leakage into the choroidal tissue must be considered as a sign of hyperpermeability of the choroidal vessels. Areas of choroidal leakage have been observed by Scheider et al. in 37\% of 19 patients with CSC, and by us, in our previous study, in $94 \%$ of 33 patients. ${ }^{9,10}$ In that first series of patients with CSC we did not find choroidal perfusion defects, which were, in contrast, seen in $63 \%$ of the cases reported by Scheider et al. ${ }^{9,10}$ The results of the study of Guyer et al. ${ }^{11}$ are in agreement with ours. In the present study we wanted to determine, in a sufficiently large number of cases, the proportion of patients with CSC who have perfusion defects and hyperpermeability alterations of the choroid. The behaviour of ICG in the choroid was evaluated in a series of cases covering the wide spectrum of clinical manifestations of CSC. In the follow-up examinations we looked for further elements which could help to reveal the role of the choroid in CSC.

We observed choroidal perfusion defects in only 4 $(2.7 \%)$ of the 145 patients in our series. Areas of dye diffusion in the choroid, on the other hand, were a practically constant finding (98.6\%). These results do not allow us to establish a causal relationship between focal delays of choroidal filling and choroidal vascular hyperpermeability in CSC. The large discrepancy between our results and those of Scheider $e t$ al. is probably due to the limited field and image resolution of the videoangiographic system (scanning laser ophthalmoscope) utilised by these authors, and to the different definition given by them to the areas of choroidal filling delay. ${ }^{9}$ Our assessment for recognising these areas was carried out in an angiographic phase advanced enough not to include physiological delays in choroidal perfusion.

As we have already discussed in our previous study, the areas of choroidal exudation, due to their shape and size, probably visualise groups of hyperpermeable lobules of the choriocapillaris. This condition of zonal hyperpermeability of the choriocapillaris seems to characterise patients with CSC in all the evolutional stages of the disease. We have found areas of choroidal leakage beneath all the alterations of the retinal pigment epithelium except for the degenerative changes secondary to chronic persistence of subretinal fluid. We have seen these areas remaining unchanged for the whole follow-up period (up to 22 months), even after regression of the neurosensory retinal detachment, if present. In 5 eyes we were able to observe that an area of hyperpermeability of the choriocapillaris preceded the appearance of leakage points on the corresponding retinal pigment epithelium. In no case did we find new areas of choroidal hyperfluorescence on the ICG angiograms performed during follow-up. All these observations induce us to believe that the abnormal permeability of the choriocapillaris in CSC precedes the alterations of the retinal pigment epithelium and presumably is the cause of these alterations. The static nature of the choroidal angiographic pattern also suggests that the causative factor of vascular hyperpermeability acts in a lasting and constant manner in patients with CSC. Another possible hypothesis is that a temporary 'noxa' may produce long-lasting or irreversible damage to the endothelium of the choriocapillaris.

The present study confirms our previous observation that in CSC diffusion and clearing of the ICG dye from the areas of hyperpermeable choriocapillaris takes place in a more evident and rapid way than in other pathological conditions in which there is leakage from abnormal choroidal vessels. ${ }^{10}$ With a large number of patients available we were also able to note a particularly rapid shifting of the dye outwards from the regions of early staining that corresponded to the pigment epithelial detachments. Beneath every detachment the dye which diffused from the abnormal choriocapillaris was cleared with a centrifugal movement in the space of a few minutes. The same pattern of diffusion of the dye in the choroidal tissue was observed in some of the areas where hypofluorescent spots appeared in the late angiographic phases. This wash-out phenomenon visualises a rapid turnover of the plasmatic proteins in the extravascular space of the choroid and owes its existence to the short duration of the choroidal recirculation phase of the ICG dye. ${ }^{16}$ 
When the concentration of ICG in the blood decreases significantly, the dye in the choroidal extravascular space originating from hyperpermeable vessels is progressively diluted and cleared.

Assuming, on the basis of the Fick Principle, that the time taken to remove a substance from a tissue is proportional to the local blood flow, we may presume a condition of elevated blood flow in the areas of choroid where ICG angiography shows the wash-out phenomenon. Using a similar theoretical basis radioactive wash-out techniques have been developed for measuring choroidal blood flow. ${ }^{17} \mathrm{We}$ propose that there is focal choroidal hyperperfusion corresponding to the areas of pigment epithelial detachment accompanying CSC. A particularly marked haemodynamic stress on the choroidal side could lead to the formation of the pigment epithelial detachments. Leaks, atrophy and hypofluorescent spots revealed by ICG angiography are the other possible responses of the retinal pigment epithelium to the exudation from the choriocapillaris.

The behaviour of ICG in the pigment epithelial detachments of CSC and the hypoffuorescent spots revealed by ICG in this disease were first described by us, ${ }^{10}$ and concurrently by Guyer et al. ${ }^{11}$ Small hypofluorescent areas surrounded by hyperfluorescent rings, corresponding to the pigment epithelial detachments, and hypofluorescent spots characterise the terminal ICG angiographic phase in many cases of CSC. With ICG angiography pigment epithelial detachments in CSC can be easily recognised and distinguished from other focal alterations of the retinal pigment epithelium. Pigment epithelial detachments were found in $27.5 \%$ of our patients and in $79 \%$ of patients with CSC studied with ICG angiography by Guyer et al. ${ }^{11}$ From these observations pigment epithelial detachments in CSC seem to be much more frequent than commonly believed. With ICG angiography the pigment epithelial detachments of CSC can also be distinguished from the serous detachments of age-related macular degeneration, which remain not fluorescent during all angiographic phases. $^{2,18}$ The different angiographic imaging characteristics could reflect a different origin of the subpigment epithelial fluid and/or different permeability conditions of Bruch's membrane. ${ }^{19}$

Hypofluorescent spots are the angiographic documentation of a pigment epithelial alteration previously not revealed by fluorescein angiography. In our series they were more evident and numerous in patients with long-lasting CSC. In one case they followed the reattachment of a pigment epithelial detachment. They did not regress in any of the patients during follow-up. In 3 eyes we saw these spots enlarge during the follow-up period, independently of the presence of subretinal or subepithelial exudation. With this finding ICG angiography offers new evidence to support the observation that alterations of the retinal pigment epithelium progress in CSC also in the absence of subretinal fluid. ${ }^{20}$ Swelling and hyperpigmentation of the pigment epithelial cells have been reported in a histological study of a serous pigment epithelial detachment accompanying CSC. ${ }^{21}$ These abnormalities could underlie the hypofluorescent lesions (pigment epithelial detachments and hypofluorescent spots) which may be found with ICG in CSC.

In conclusion, the results of this study provide positive support for the concept, already expressed by Gass ${ }^{14}$ many years ago and recently taken up again by our group and other authors, ${ }^{9-11}$ that choroidal exudation precedes pigment epithelial alterations in CSC. For serous detachment to form in CSC the rate of exudation into the subretinal space must exceed the rate of active and passive fluid removal through the retinal pigment epithelium. ${ }^{22} \mathrm{~A}$ high rate of subretinal influx or an abnormally low capacity for reabsorption of the subretinal fluid could alternatively be considered as the causative factor for accumulation of fluid. On the basis of experimental data Marmor ${ }^{23,24}$ suggests that impairment of the transport mechanisms of the retinal pigment epithelium around the site of leakage is a necessary condition in CSC. A functional alteration of the retinal pigment epithelium may be supposed in the areas where ICG angiography reveals damage to the choriocapillaris. However, we think that massive exudation originating from an area with abnormal permeability of the choriocapillaris could reach the subretinal space with a rate of flow so high as to overwhelm also the normal transport mechanisms of the retinal pigment epithelium. On the basis of our observation we also propose that when the zonal hyperpermeability of the choriocapillaris corresponds to a local condition of particularly elevated blood flow, retinal pigment epithelial detachments can occur.

This study was presented in part at the Third Meeting of the European Macula Group, Athens, Greece, 20-22 April 1994, and at the International Symposium on Fluorescence Angiography, Quebec City, Canada, 20-24 June 1994.

Key words: Central serous chorioretinopathy, Fluorescein angiography, Indocyanine green angiography, Retinal pigment epithelium, Subretinal fluid.

\section{REFERENCES}

1. Scheider A, Schroedal C. High resolution indocyanine green angiography with scanning laser ophthalmoscope. Am J Ophthalmol 1989;108:458-9.

2. Yannuzzi LA, Slakter JS, Sorenson JA, Guyer DR, Orlock DA. Digital indocyanine green videoangiography and choroidal neovascularization. Retina 1992; 12:191-223.

3. Cardillo Piccolino F, Borgia L, Zingirian M. Indocyanine green choroidal videoangiography during induced 
intraocular hypertension. Am J Ophthalmol 1993;115: 817-8.

4. Slakter JS, Yannuzzi LA, Sorenson JA, Guyer DR, Ho AC, Orlock DA. A pilot study of indocyanine green videoangiography-guided laser photocoagulation of occult choroidal neovascularization in age-related macular degeneration. Arch Ophthalmol 1994;112: 465-71.

5. Gass JDM. Idiopathic central serous chorioretinopathy. In: Stereoscopic atlas of macular diseases: diagnosis and treatment. 3rd ed. St Louis, MO: CV Mosby, 1987:45-59.

6. Spitznas M. Pathogenesis of central serous retinopathy: a new working hypothesis. Graefes Arch Clin Exp Ophthalmol 1986;224:321-4.

7. Marmor MF. New hypothesis on the pathogenesis and treatment of serous retinal detachment. Graefes Arch Clin Exp Ophthalmol 1988;226:548-52.

8. Hayashi K, Hasegawa Y, Tokoro T. Indocyanine green angiography of central serous chorioretinopathy. Int Ophthalmol 1986;9:37-41.

9. Scheider A, Nasemann JE, Lund OE. Fluorescein and indocyanine green angiography of central serous chorioretinopathy by scanning laser ophthalmoscopy. Am J Ophthalmol 1993;115:50-6.

10. Cardillo Piccolino F, Borgia L. Central serous chorioretinopathy and indocyanine green angiography. Retina 1994;14:231-42.

11. Guyer DR, Yannuzzi LA, Slakter JS, Sorenson JA, Ho A, Orlock D. Digital indocyanine green angiography of central serous chorioretinopathy. Arch Ophthalmol 1994;112:1057-62.

12. Jalk AE, Jabbour N, Avila MP, Trempe CL, Schepens CL. Retinal pigment epithelium decompensation. I. Clinical features and natural course. Ophthalmology 1984;91:1544-8.

13. Yannuzzi LA, Shakin J, Fisher Y, Altomonte MA. Peripheral retinal detachments and retinal pigment epithelial atrophic tracks secondary to central serous pigment epitheliopathy. Ophthalmology 1984;91: 1554-72.

14. Gass JDM. Pathogenesis of disciform detachment of the neuroepithelium. II. Idiopathic central serous choroidopathy. Am J Ophthalmol 1967;63:587-92.

15. Cherrick GR, Stein SW, Leevy CM, Davidson CS. Indocyanine green: observations on its physical properties, plasma decay, and hepatic extraction. J Clin Invest 1960;39:592-7.

16. Paumgartner $G$. The handling of indocyanine green by the liver. Schweiz Med Wochenschr 1975;105 (Suppl.17):1-30.

17. Strang R. Physical factors in the measurements of blood flow by radioactive washout technique. In: Cont JS, editor. Vision and circulation. London: Henry Kimpton, 1976:39-44.

18. Yannuzzi LA, Hope-Ross M, Slakter JS, Guyer DR, Sorenson JA, Ho AC, Sperber DE, Freund KB, Orlock DA. Analysis of vascularised pigment epithelial detachments using indocyanine green videoangiography. Retina 1994;14:99-113.

19. Bird AC, Marschall J. Retinal pigment epithelial detachments in the elderly. Trans Ophthalmol Soc UK 1986;105:674-82.

20. Levine R, Brucker AJ, Robinson F. Long-term followup of idiopathic central serous chorioretinopathy by fluorescein angiography. Ophthalmology 1989;96: 854-9.

21. Mori N. Serous detachment of the retinal pigment epithelium: histologic study on central serous retinopathy with pigment epithelial detachment. Jpn. J Clin Ophthalmol 1972;26:259-63.

22. Cardillo Piccolino F. Central serous chorioretinopathy: some considerations on the pathogenesis. Ophthalmologica 1981;182:204-10.

23. Marmor MF. Control of subretinal fluid: experimental and clinical studies. Eye 1990;4:340-4.

24. Marmor MF, Yao XY. Conditions necessary for the formation of serous detachment. Arch Ophthalmol 1994;112:830-8. 